\title{
Acupuncture for Treatment of Erectile Dysfunction: A Systematic Review and Meta-Analysis
}

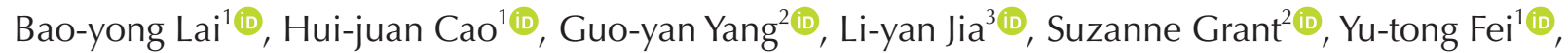 \\ Emma Wong ${ }^{2}$ (i), Xin-lin $\mathrm{Li}^{1}{ }^{(\mathbb{D})}$, Xiao-ying Yang ${ }^{1}$ (i), Jian-ping Liu ${ }^{1,4}$ \\ ${ }^{1}$ Centre for Evidence-Based Chinese Medicine, Beijing University of Chinese Medicine, Beijing, China, ${ }^{2}$ NICM Health Research Institute, \\ Western Sydney University, Penrith, Australia, ${ }^{3}$ School of Traditional Chinese Medicine, Beijing University of Chinese Medicine, Beijing, \\ China, ${ }^{4}$ Institute of Integrated Traditional Chinese Medicine and Western Medicine, Guangzhou Medical University, Guangzhou, China
}

Purpose: To assess the effectiveness and safety of acupuncture for erectile dysfunction (ED).

Materials and Methods: We searched six major English and Chinese databases included randomized controlled trials (RCTs) testing acupuncture alone or in combination for ED. Dichotomous data were presented as risk ratio (RR) and continuous data were presented as mean difference (MD) both with 95\% confidence interval (CI). The Revman (v.5.3) was used for data analyses. Quality of evidence across studies was assessed by the online GRADEpro tool.

Results: We identified 22 RCTs, fourteen of them involving psychogenic ED. Most of the included RCTs had high or unclear risk of bias. There was no difference between electro-acupuncture and sham acupuncture with electrical stimulation on the rate of satisfaction and self-assessment ( $R R, 1.50 ; 95 \% \mathrm{Cl}, 0.71-3.16 ; 1$ trial). Acupuncture combined with tadalafil appeared to have better effect on increasing cure rate (RR, $1.31 ; 95 \% \mathrm{Cl}, 1.00-1.71 ; 2$ trials), and International Index of Erectile Function-5 scores (MD, 5.38; 95\% Cl, 4.46-6.29; 2 trials). When acupuncture plus herbal medicine compared with herbal medicine alone, the combination therapy showed significant better improvement in erectile function $(\mathrm{RR}, 1.68 ; 95 \% \mathrm{Cl}, 1.31-2.15$; 7 trials). Only two trials reported facial red and dizziness cases, and needle sticking and pruritus cases in acupuncture group. Conclusions: Low quality evidence shows beneficial effect of acupuncture as adjunctive treatment for people mainly with psychogenic ED. Safety of acupuncture was insufficiently reported. The findings should be confirmed in large, rigorously designed and well-reported trials.

Keywords: Acupuncture; Erectile dysfunction; Meta-analysis; Randomized controlled trial; Systematic review

This is an Open Access article distributed under the terms of the Creative Commons Attribution Non-Commercial License (http://creativecommons.org/licenses/by-nc/4.0) which permits unrestricted non-commercial use, distribution, and reproduction in any medium, provided the original work is properly cited.

\section{INTRODUCTION}

Erectile dysfunction (ED), also called impotence, is defined as an inability to obtain or maintain a penile erection sufficient for satisfactory sexual intercourse
[1]. ED is a common clinical condition, affecting men of all ages, particularly the elderly. ED affects around $52 \%$ in men aged 40 to 70 years, with more than 320 million men predicted to suffer from ED by 2025 years worldwide [2]. ED can result in considerable distress

Received: Oct 7, 2018 Revised: Jan 18, 2019 Accepted: Feb 4, 2019 Published online Mar 15, 2019

Correspondence to: Jian-ping Liu (iD https://orcid.org/0000-0002-0320-061X

Centre for Evidence-Based Chinese Medicine, Beijing University of Chinese Medicine, No. 11, Bei San Huan Dong Lu, Chaoyang District, Beijing 100029, China.

Tel: +86-10-64286760, Fax: +86-10-64286871, E-mail: Liujp@bucm.edu.cn 
and lowered quality of life. As it is associated with a wide variety of underlying conditions such as diabetes and cardiovascular co-morbidities, treatment options depend upon the associated factors and diverse approaches in different settings [3]. Current treatment for ED included oral drugs, intrapenile therapies and penile prosthesis implantation, but with uncertain effect [4]. Alternative options such as non-pharmaceutical therapies are needed and expected.

Acupuncture, part of traditional Chinese medicine (TCM), is increasingly used for the treatment of ED. There are different ways for its use, including stimulation of acupoints with needling, heating (moxibustion), electrical current, or injecting drugs into acupoints [5]. TCM theory states that ED is usually caused by the decline of fire from the life gate (Ming men), sexual indulgence or frequent masturbation, and emotional disturbances. Thus, the principle of acupuncture treatment is to invigorate the kidney $q i$ and nourish the heart and spleen. As such, and selecting acupoints from the Kidney Meridian of Foot-Shaoyin and the Ren Meridian and back-shu points is commonly used for treating ED, and moxibustion can be used as well during the treatment [6,7].

The potential mechanism of action by how acupuncture may have an effect on ED is unclear. However, there is some indication that acupuncture may stimulate nerve endings, and induce nerve impulses which then impact on levels of norepinephrine, acetylcholine, and their biological enzymes in the central nervous system [8]. Some clinical trials have been conducted to investigate the effect of acupuncture in the treatment of ED. A systematic review published in 2016 concluded with insufficient results about the effect of acupuncture when comparing with sham acupuncture and psychological therapy [9]. Another recent systematic review summarized evidence of acupuncture for ED [10]. However, this review searched literature mainly from Chinese databases, and its control groups were only Chinese herbal medicine. As a result, the interpretation of the findings may be limited. Our review aims to comprehensively review the current evidence of acupuncture for ED.

\section{MATERIALS AND METHODS}

\section{Inclusion/exclusion criteria}

We included both parallel, cross-over, randomized clinical trials, regardless of blinding and publication status. Types of participants included men who were diagnosed as ED by any recognized national or international criteria, regardless of psychogenic and organic origin of impotence. Interventions included as verum acupuncture (defined as needling stimulation of acupuncture points or trigger points by manual acupuncture with or without heating [moxibustion]), electro-stimulating, acupoint injection, acupressure and laser acupuncture [5,6]. Controls included no treatment, sham acupuncture, herbal medicine, or conventional medicine. Co-interventions were allowed as long as they were given equally to all randomized arms.

\section{1) Primary outcomes}

(1) Patient erectile function and partner satisfaction measured by International Index of Erectile Function-5 (IIEF-5) score and its components [11]; (2) The effect and quality of sexual intercourse presented as "cure", or "markedly improved on erectile function" based on validated measurement tools or scales. "Cure" was defined as symptom disappearance with successful sexual activity and/or with IIEF-5 score $\geq 22$ [11]. "Markedly improved on erectile function" referred to that all the three below items were met: the self-report disappearance of clinical symptoms, the erection angle of penis is more than 90 degrees in sexual activity, and the success rate of sexual intercourse is over $75 \%$.

\section{2) Secondary outcomes}

(1) The quality of sexual activity measured by "satisfaction and self-assessment", which defined as the self-reported satisfaction by patients or their partners. They reported that the symptoms disappeared, at same time, erectile function and sexual life returned to normal; (2) Angle of penile erection measured by selfassessment tools or scales; (3) Adverse events.

\section{Search strategy}

We searched for published studies in two English and four Chinese electronic databases from their inception to August 31st, 2018, including PubMed, the Cochrane Library, Sinomed Database, China National Knowledge Infrastructure, Wanfang Database, and China Science Technology Journal Database. The search terms included acupuncture-related terms (i.e., "acupuncture", "electro-acupuncture", "auricular therapy", "warm needling”, "fire needling”, "shark hook 
needling", "magnet needle", "acupoint injection”, "point injection", "moxibustion", "acupressure”), combined with erectile dysfunction related terms (i.e., "erectile dysfunction [Mesh terms]", "impotence", "yang wei"). Search term strategies were adapted for each specific database.

\section{Study selection and data extraction}

Two authors (Lai BY and Jia LY) independently selected the trials included in the review according to the inclusion/exclusion criteria. Any disagreement was resolved by discussion. We performed data extraction using a self-developed data extraction form. If the necessary data were not available in the trial reports, further information was sought by contacting corresponding author.

\section{Assessment of methodological quality}

The risk of bias of the included trials was assessed independently according to the criteria from the Cochrane Handbook for Systematic Reviews of Interventions [12]. Criteria included adequacy of generation of the allocation sequence, allocation concealment, blinding (blinding of participants and personnel, blinding of outcome assessors), incomplete outcome data or not, whether selected reporting the results and other bias (e.g., imbalance of the baseline information). Risk of bias for each trial was assessed as low, high, or unclear. A trial was considered as having low risk of bias when all the items met the criteria; a trial was considered at high risk of bias when at least one of the items was not met; and a trial was considered unclear risk of bias where insufficient information was available to make the judgment. Any difference in the quality assessment of trials was resolved by discussion in order to reach consensus. Quality of evidence across studies for each important outcome was assessed using the online GRADE approach to support the recommendations using the online GRADEpro tool (https://gradepro.org/).

\section{Data analysis}

Meta-analysis was performed within comparisons of the same type of acupuncture versus the similar control. Dichotomous data were presented as risk ratio (RR) and continuous outcomes as mean difference (MD), both with $95 \%$ confidence intervals (CI). We used $I$ square value to detect statistical heterogeneity and to measure the percentage of the variability in effect sizes between studies that is due to heterogeneity rather than to sampling error. We used random effects model to combine the results in this review due to potential sources of clinical heterogeneity [12]. If the $I^{2}>75 \%$, we did not pool the data and results from each individual trial were presented respectively. The statistical analysis was carried out using Revman 5.3 software. If a sufficient numbers of randomized trials were identified and data available, subgroup analysis would be performed according to the comparisons.

\section{RESULTS}

\section{Study selection}

We identified 300 studies, of which 68 duplicates were removed. After screening the abstracts, 190 trials

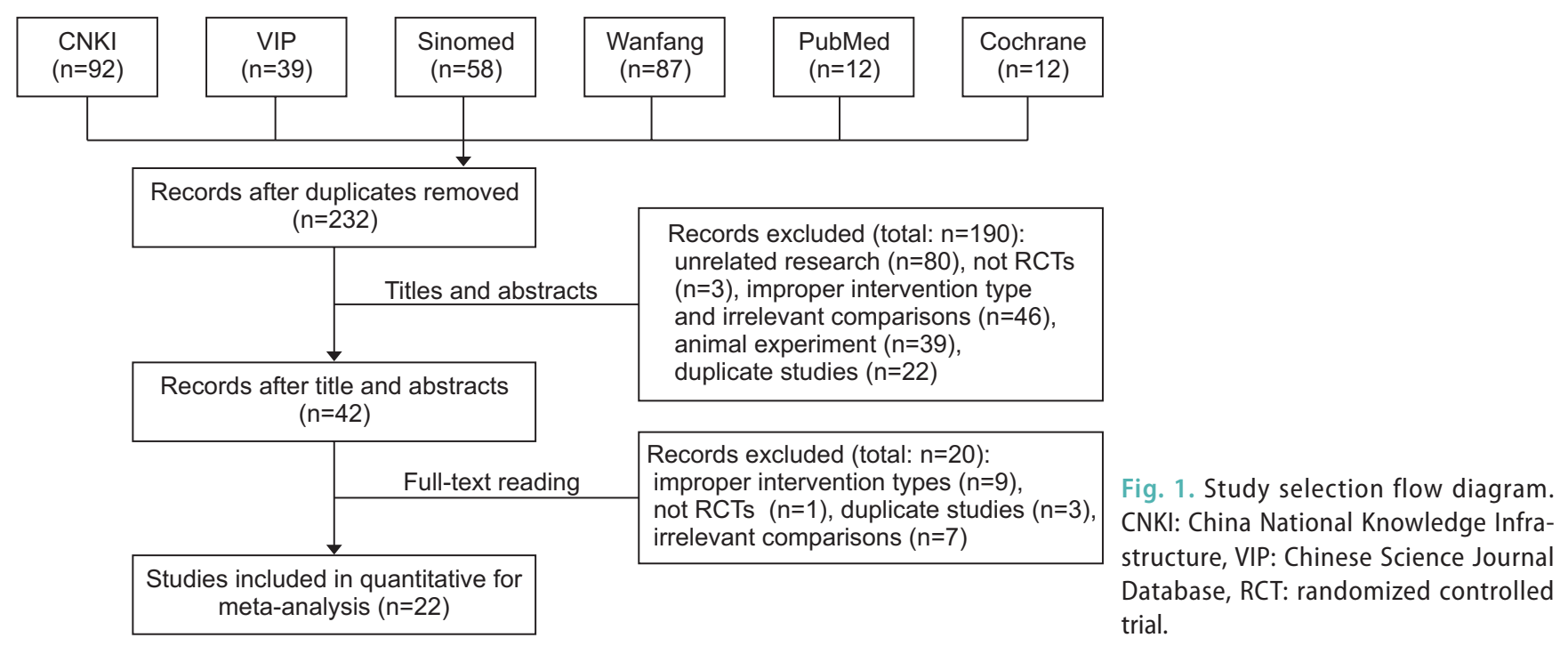


were excluded with reasons. Full-texts of the remaining 42 trials were retrieved and assessed for eligibility. A total of 22 randomized controlled trial (RCT) met the inclusion criteria. Fig. 1 illustrates a PRISMA flow diagram.

\section{Description of studies}

Twenty-two RCTs involving 1,751 participants were enrolled in this review. All of them were conducted in China, and 2 of them published in English. All trials had parallel comparisons, except one [13] that used a crossover design. The sample size varied from 21 to 176 participants, with an average of 35 patients per group. The participants were male adults aged from 20 to 69 years old. The interventions included manual acupuncture with or without moxibustion, electro-acupuncture, and acupuncture point injection. Type of controls included sham acupuncture, herbal medicine, Western medicine, psychological therapy, and hypnosis therapy. The types of ED of twenty-two trials included 2 trials $[14,15]$ were involving ED participants with type-2 diabetes, two trials [16,17] were involving ED participants with psychogenic or arterial supply insufficiency, fourteen trials [13,18-30] were ED participants with psychogenic, and four trials [31-34] not reported ED information. The characteristics of included studies are shown in Table 1.

\section{Description of therapeutic regimen}

A summary of acupuncture regimen is provided in Appendix, which contains a list of acupoints, treatment frequency and duration for each trial (Appendix). Participants of included trials accepted acupuncture one session daily for 30 minutes at most of the occasions. The principle of acupuncture treatment was to improve the erectile function and the most common used acupoints and involving meridian were Guan Yuan (CV4, the Ren Meridian, 15 trials), San Yin Jiao (SP6, the Splenic Meridian of Foot-taiyin, 15 trials), Shen Shu (BL23, the Bladder Meridian Foot-taiyang, 13 trials), $Z u$ San $L i$ (ST36, the Stomach Meridian of Foot-yangming, 12 trials), Ming Men (DU4, the Du Meridian of Foot-shaoyin, 8 trials), Tai Chong (LR3, the Liver Meridian of Foot-jueyin, 7 trials), Tai Xi (KI3, the Kidney Meridian of Foot-shaoyin, 7 trials) and $C i$ Liao (BL32, the Bladder Meridian Foot-taiyang, 5 trials).

\section{Assessment of risk of bias}

According to the pre-defined approach, 22 trials were found to be either unclear $(n=19)$ or high risk of bias $(n=3)$ due to insufficient or inadequate reporting of the information. Only 6 trials [14,16,19,25,27,30] described that random number table was used to generate the random allocation, thus assessed as having low risk of bias. However, none of the trials reported the allocation concealment. Due to special characteristics of acupuncture, it is difficult for blinding to practitioners, so only two trials $[13,18]$ used sham acupuncture as control and were assessed as low risk of performance bias regarding to the potential adequate blinding method of participants. Risk of detective bias was assessed to be unclear since none of them reported the method of blinding to outcome assessors or statistician. One trial [18] reported number and reasons for drop-out participants, which was regarded low risk of attrition bias. The other 21 trials did not specify the drop-out, and were all evaluated as unclear risk of attrition bias. Twenty trials were assessed as having unclear risk of selective reporting bias due to the absence of protocol; the remaining three trials $[22,25,26]$ were assessed as high risk of selective reporting bias since they all had obvious problems on primary outcome reporting. Other bias was assessed by comparability between groups on baseline data such as age and duration of ED. Only six trials $[16,18,21,24,31,32]$ reported baseline data including age and duration of dysfunction. There is no statistical description of details, so the risk of bias was assessed as unclear. The methodological quality of all the included trials is shown in the Fig. 2.

\section{Effect estimates}

Data analysis was conducted according to the type of comparisons. Table 2 illustrates the details of effect estimates of acupuncture for ED.

\section{1) Acupuncture versus sham acupuncture}

One trial [18] compared electro-acupuncture with sham acupuncture (needles inserted into non acupoints), the results showed no difference on the rates of satisfaction and self-assessment between groups (RR, 1.50; 95\% CI, 0.71-3.16; 60 participants). Another crossover trial [13] compared manual acupuncture with sham acupuncture (needle insert into irreverent points) showed significant better effect on the rates of satisfaction and self-assessment (RR, 7.53; 95\% CI, 1.13-50.00; 


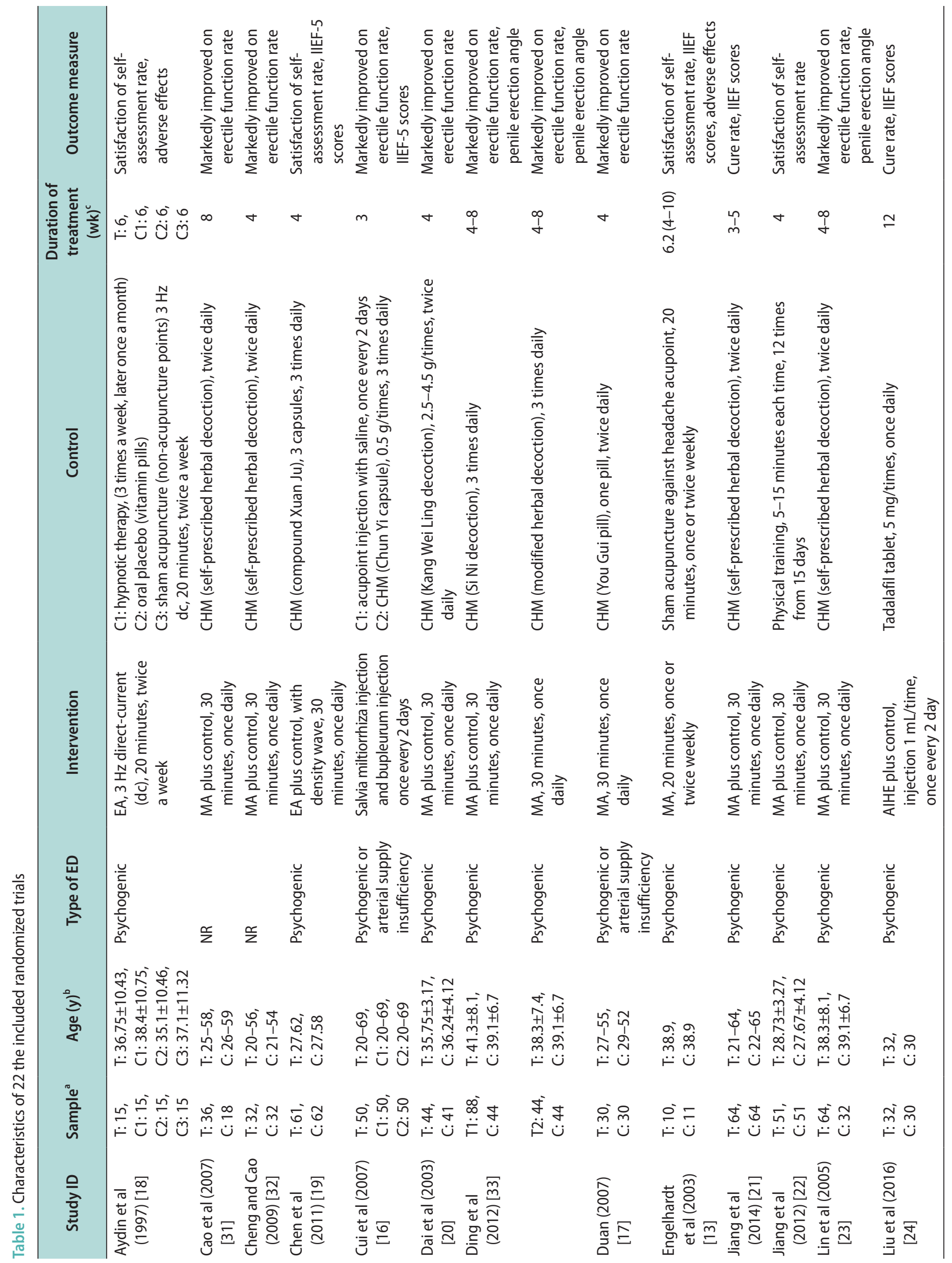




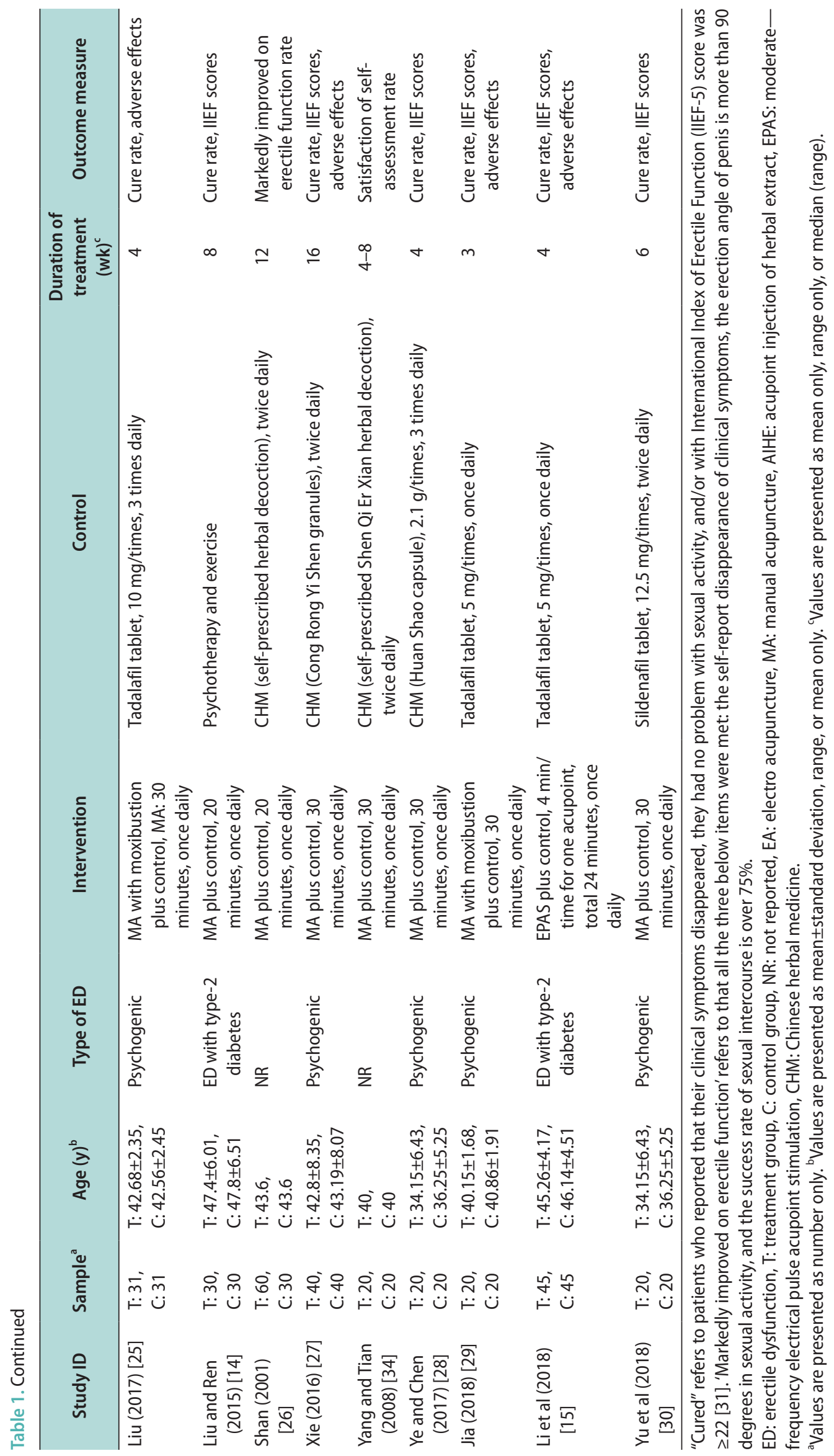




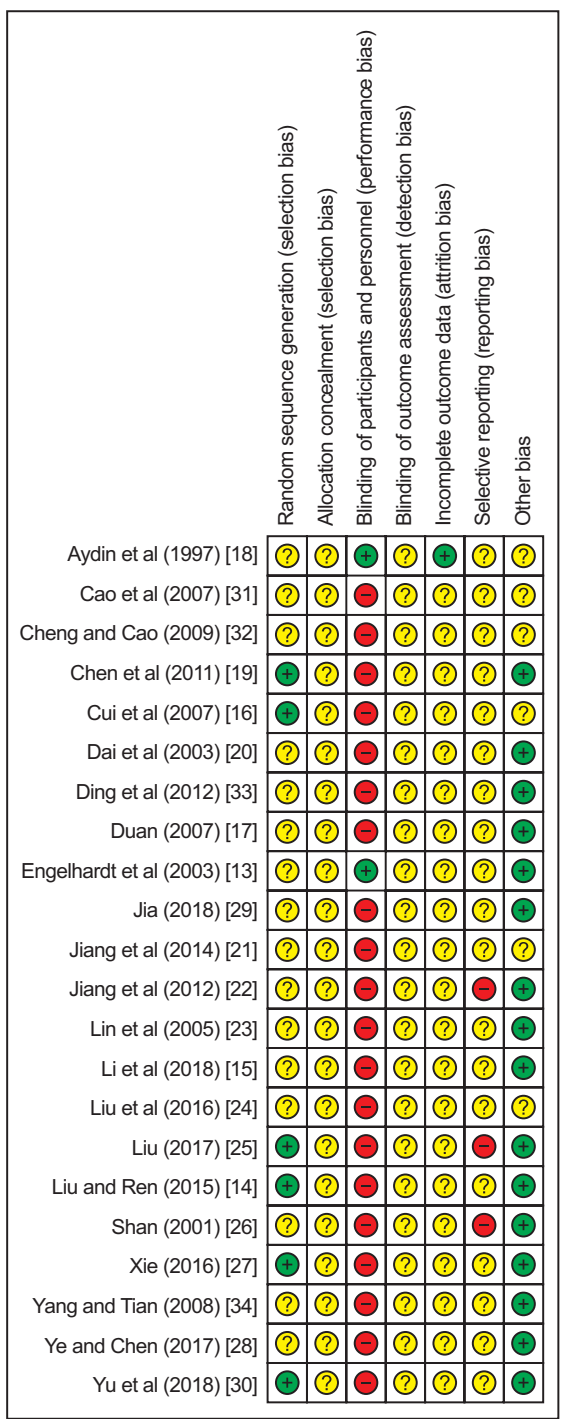

Fig. 2. Risk of bias summary.

60 participants). No other results were reported from these two trials.

\section{2) Acupuncture plus western medicine versus western medicine}

Two trials $[15,24]$ compared acupuncture plus tadalafil compared with tadalafil alone. The combination therapy showed better effect on increasing cure rate $\left(\mathrm{RR}, 1.31 ; 95 \% \mathrm{CI}, 1.00-1.71 ; I^{2}=0 \% ; 2\right.$ trials; 152 participants) and IIEF-5 scores (MD, 5.38; 95\% CI, 4.46-6.29; $I^{2}=0 \%$; 2 trials; 152 participants). Another trial [30] compared acupuncture plus sildenafil compared with sildenafil alone, and the combination therapy showed better effect on increasing IIEF-5 scores (MD, 3.23; 95\% CI, 2.12-4.34; 1 trial; 70 participants). There was no significant between-group difference in terms of cure rate
(RR, 3.0; 95\% CI, 0.65-13.86; 1 trial; 70 participants).

\section{3) Manual acupuncture plus moxibustion versus tadalafil tablet}

Two trials $[25,29]$ compared acupuncture plus moxibustion compared with tadalafil alone. There was no significant difference between groups in cure rate $(R R$, 1.40; 95\% CI, 0.74-2.66; 2 trials; $I^{2}=0 \% ; 102$ participants). No difference between groups was found from one trial [29] in IIEF-5 scores (MD, 1.15; 95\% CI, 1.37-0.93; 40 participants).

\section{4) Acupuncture versus herbal medicine}

Two trials [17,33] compared acupuncture with herbal medicine and another trial [16] compared acupoint injection of herbal extracts with oral herbal medicine. There was no difference between acupuncture and herbal medicine in terms of markedly improved on erectile function rate (RR, 1.40; 95\% CI, 0.42-4.69; $I^{2}=46 \%$; 2 trials; 148 participants) [17,33]. However, the acupoint injection of herbal extracts significantly increased the IIEF-5 scores compared to oral herbal medicine (MD, 4.0; 95\% CI, 3.66-4.34; 1 trial; 100 participants) [16].

\section{5) Acupuncture plus herbal medicine versus herbal medicine alone}

Acupuncture plus herbal medicine was tested in seven trials [20,23,26,27,31-33], which showed significant better effect on markedly improved rate on erectile function (as measured by the erection angle and success rate of sexual intercourse) than herbal medicine alone (RR, 1.68; 95\% CI, 1.31-2.15; $I^{2}=0 \%$; 7 trials; 601 participants).

By excluding three trials [26,31,32] for not reporting types information of $\mathrm{ED}$, remaining four trials only involving ED patients with psychogenic also show that better effect on markedly improved rate on erectile function in combination therapy group (RR, 1.63; 95\% CI, 1.21-2.19; $I^{2}=0 \%$; 4 trials; 393 participants). The pooled results also showed significantly better effects of the combination therapy on cure rate $(\mathrm{RR}, 1.36 ; 95 \%$ CI, $1.12-1.65, I^{2}=0 \%$; 2 trials; 168 participants) [21,28] and rigidity (as measured by erectile angle) in sexual intercourse (MD, 6.73 degree; 95\% CI, 4.10-9.36; $I^{2}=0 \% ; 2$ trials; 228 participants) [23,33]. However, no difference was found between groups in the rate of satisfaction and self-assessment (RR, 1.67; 95\% CI, 0.64-4.36; $I^{2}=75 \%$; 
을

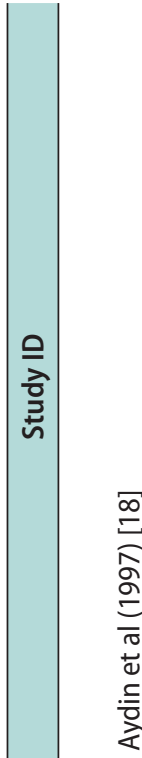

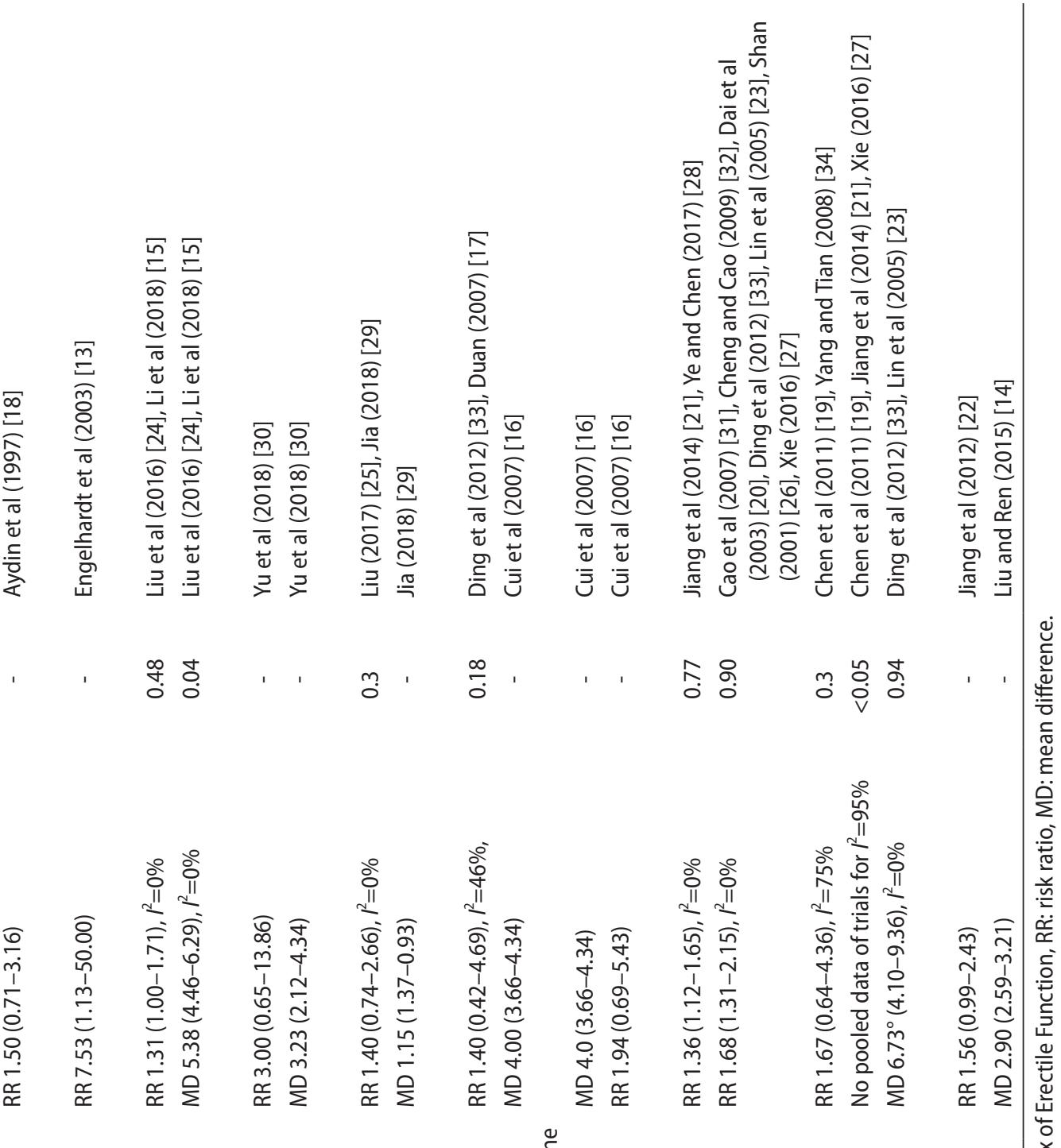
든<smiles>[13CH][13CH]</smiles>

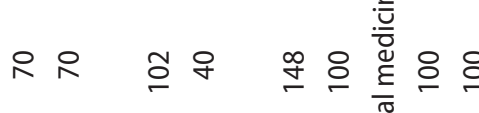

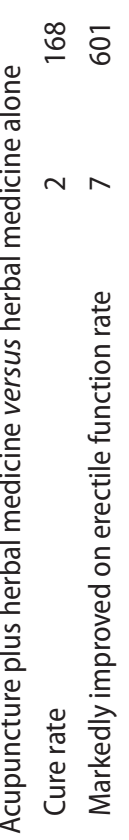

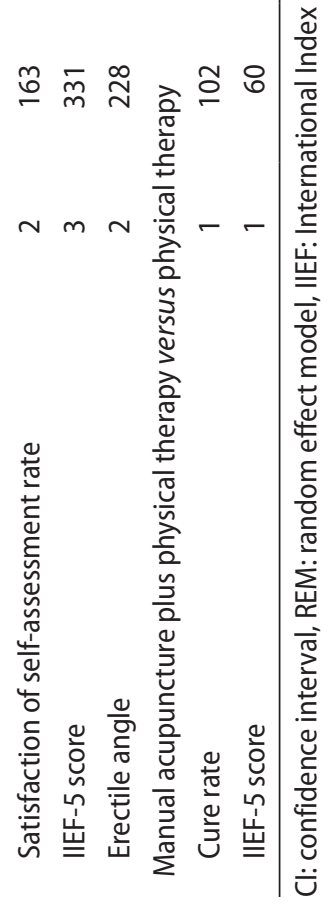


2 trials; 163 participants) [19,34]. Moreover, three trials $[19,21,27]$ favored the combination therapy of acupuncture and herbal medicine in higher IIEF-5 scores (MD, 3.53) to herbal medicine alone, but the results could not be pooled due to high statistical heterogeneity $\left(I^{2}=97 \%\right)$.

\section{6) Acupuncture plus psychological therapy versus psychological therapy}

Two trials compared acupuncture plus psychological therapy with psychological therapy alone. One of them [22] found no difference between groups for cure rate (RR, 1.56; 95\% CI, 0.99-2.43; 1 trial; 102 participants). Another trial [14] involving 60 ED participants with type-2 diabetes and found the combination therapy appeared to be better on higher IIEF-5 scores (MD, 2.90; 95\% CI, 2.59-3.21; 1 trial; 60 participants).

\section{Adverse events}

Seven trials reported the outcome of adverse events and side effects $[13,15,16,18,25,27,29]$. One trial [16] reported five cases of dyspepsia, two cases of dizziness and one case of dry mouth from tadalafil in the control group; and one case having facial red and one case of dizziness in acupuncture group. Another one trial [29] reported three patients suffered from needle sticking and pruritus during treatment in acupuncture group; and there were two cases having dry mouth and headache from tadalafil in control group. No adverse effect or side effect was found in the remaining 5 trials.

\section{Overall quality of evidence by GRADE}

We graded the overall quality of available evidence using GRADE criteria. When combination of acupuncture compared to tadalafil, the quality of evidence for cure rate and IIEF-5 scores was low. In comparison of other interventions and outcome assessments, the quality of evidence was mainly low or very low due to high risk of bias, imprecision (small number of total events or small sample size), or indirectness (outcome measures). Detail of result is show in Table 3.

\section{Additional analysis}

We tried to do the subgroup analyses according to the types of ED participants, but failed, due to the insufficient number of trials and related information. We could not perform other meaningful sensitivity analysis either.

\section{DISCUSSION}

\section{Summary of the main results}

Twenty-three RCTs were included in this review. The majority of the included studies were having high or unclear risk of bias. Low quality evidence showed there was no difference between acupuncture alone and sham acupuncture, tadalafil, or herbal medicine on symptoms improvement for ED. Combination of acupuncture appeared to show beneficial effect of acupuncture as adjunctive treatment for ED participants with psychogenic or partly with type-2 diabetes, when compared with other conventional therapies (such as tadalafil, psychological therapy or herbal medicine). However, the level of evidence for all outcomes were assessed as "low" or "very low" due to high risk of bias, imprecision or indirectness among included trials. The findings need to be seen as inconclusive due to small sample size and poor methodological quality. Safety of acupuncture was insufficiently reported in the included trials.

\section{Comparison with previous studies}

There are two published reviews on this topic. One included 3 RCTs involving 183 participants with ED [9], which compared acupuncture with sham acupuncture and psychological therapy respectively. The included RCTs in this review failed to show a specific therapeutic effect of acupuncture, and had methodological flaws as concluded by the authors. And the other recent one only involved Chinese database and included 6 RCTs, control treatment were only involving Chinese herbal medicine and details of treatment information were not clearly specified [10]. In addition, the findings of both two reviews of included trials were not finally pooled due to statistical and clinical heterogeneity, which failed to show a specific therapeutic effect of acupuncture for ED. Compared to the previous two reviews, this update review covered a broader combination of studies, including acupuncture with moxibustion, the acupoint injection and different comparisons, and additional outcome assessments. We performed analyses based on different comparisons and found that although the strength of the evidence was weak, the findings showed there was a potential add-on effect of acupuncture for patients with ED. A total of 31.8\% (7/22) of included trials had reported adverse information from acupuncture. This review provided latest evi- 
Table 3. Summary of main findings of RCTs on acupuncture for erectile dysfunction

\begin{tabular}{|c|c|c|c|c|c|}
\hline \multirow[b]{2}{*}{ Outcome } & \multirow{2}{*}{$\begin{array}{c}\text { No. of } \\
\text { participant } \\
\text { (No. of RCT) }\end{array}$} & \multirow{2}{*}{$\begin{array}{l}\text { Quality of the } \\
\text { evidence }\end{array}$} & \multirow{2}{*}{$\begin{array}{l}\text { Relative effect } \\
\qquad(95 \% \mathrm{Cl})\end{array}$} & \multicolumn{2}{|c|}{ Anticipated absolute effect } \\
\hline & & & & $\begin{array}{l}\text { Risk with } \\
\text { control }\end{array}$ & $\begin{array}{l}\text { Risk difference with } \\
\text { intervention }(95 \% \mathrm{Cl})\end{array}$ \\
\hline \multicolumn{6}{|c|}{ Electronic acupuncture versus sham acupuncture } \\
\hline Satisfaction of self-assessment rate & $30(1)$ & $\bigoplus \bigcirc \bigcirc \bigcirc^{\text {acd }}$ & RR $1.50(0.71-3.16)$ & 400 per 1,000 & $\begin{array}{l}200 \text { more per } 1,000 \text { ( } 116 \text { more } \\
\text { to } 864 \text { more) }\end{array}$ \\
\hline \multicolumn{6}{|c|}{ Manual acupuncture versus sham acupuncture } \\
\hline Satisfaction of self-assessment rate & $30(1)$ & $\oplus \bigcirc \bigcirc \bigcirc^{\text {acd }}$ & RR $7.53(1.13-50.00)$ & 91 per 1,000 & $\begin{array}{l}594 \text { more per } 1,000 \text { ( } 12 \text { more to } \\
1,000 \text { more) }\end{array}$ \\
\hline \multicolumn{6}{|c|}{ Acupuncture plus tadalafil tablet versus tadalafil tablet } \\
\hline Cure rate & $152(2)$ & $\oplus \oplus \bigcirc \bigcirc^{\mathrm{ac}}$ & RR $1.31(1.00-1.71)$ & 467 per 1,000 & $\begin{array}{l}145 \text { more per } 1,000 \text { ( } 0 \text { more to } \\
331 \text { more) }\end{array}$ \\
\hline IIEF-5 score & $152(2)$ & $\oplus \oplus \bigcirc \bigcirc^{\mathrm{ac}}$ & $\mathrm{N} / \mathrm{A}$ & & $\begin{array}{l}\text { MD } 5.38 \text { higher ( } 4.46 \text { higher to } \\
6.29 \text { higher) }\end{array}$ \\
\hline \multicolumn{6}{|l|}{ Acupuncture versus herb medicine } \\
\hline $\begin{array}{l}\text { Markedly improved on erectile } \\
\text { function rate }\end{array}$ & $204(3)$ & $\oplus \bigcirc \bigcirc \bigcirc^{\text {acd }}$ & RR $1.51(0.96-2.38)$ & 194 per 1,000 & $\begin{array}{l}99 \text { more per } 1,000 \text { (8 fewer to } \\
268 \text { more) }\end{array}$ \\
\hline IIEF-5 score & $100(1)$ & $\oplus \oplus \bigcirc \bigcirc^{\mathrm{ac}}$ & N/A & & $\begin{array}{l}\text { MD } 4 \text { higher ( } 3.66 \text { higher to } 4.34 \\
\text { higher) }\end{array}$ \\
\hline \multicolumn{6}{|c|}{ Acupuncture plus herb medicine versus herb medicine } \\
\hline Cure rate & $168(2)$ & $\bigoplus \oplus \bigcirc \bigcirc^{\mathrm{ac}}$ & RR $1.36(1.12-1.65)$ & 607 per 1,000 & $\begin{array}{l}219 \text { more per 1,000 (73 more to } \\
395 \text { more) }\end{array}$ \\
\hline $\begin{array}{l}\text { Markedly improved on erectile } \\
\text { function rate }\end{array}$ & $601(7)$ & $\oplus \bigcirc \bigcirc \bigcirc^{\text {acd }}$ & RR $1.68(1.31-2.17)$ & 241 per 1,000 & $\begin{array}{l}164 \text { more per 1,000 (75 more to } \\
281 \text { more) }\end{array}$ \\
\hline Satisfaction of self-assessment rate & $163(2)$ & $\bigoplus \bigcirc \bigcirc \bigcirc^{\mathrm{abc}}$ & RR $1.67(0.64-4.36)$ & 463 per 1,000 & $\begin{array}{l}310 \text { more per } 1,000 \text { ( } 167 \text { fewer } \\
\text { to } 1,557 \text { more) }\end{array}$ \\
\hline IIEF-5 score & $331(3)$ & $\oplus \oplus \bigcirc \bigcirc^{\mathrm{ab}}$ & $\mathrm{N} / \mathrm{A}$ & & $\begin{array}{l}\text { MD } 3.53 \text { higher ( } 0.65 \text { higher to } \\
6.4 \text { higher) }\end{array}$ \\
\hline Erectile angle & $228(2)$ & $\oplus \bigcirc \bigcirc \bigcirc^{\text {acd }}$ & $\mathrm{N} / \mathrm{A}$ & & $\begin{array}{l}\text { MD } 6.73 \text { higher ( } 4.1 \text { higher to } \\
\text { 9.36 higher) }\end{array}$ \\
\hline
\end{tabular}

GRADE Working Group grades of evidence. High quality: We are very confident that the true effect lies close to that of the estimate of the effect. Moderate quality: We are moderately confident in the effect estimate: the true effect is likely to be close to the estimate of the effect, but there is a possibility that it is substantially different. Low quality: Our confidence in the effect estimate is limited: the true effect may be substantially different from the estimate of the effect. Very low quality: We have very little confidence in the effect estimate: the true effect is likely to be substantially different from the estimate of effect.

RCT: randomized controlled trial, Cl: confidence interval, IIEF: International Index of Erectile Function, RR: risk ratio, N/A: not applicable, MD: mean difference.

${ }^{\text {a }}$ Risk of bias: All the trials had high risk of performance bias for not blinding the participants. Methodological quality of these trials was graded as "high risk of bias," due to the design of comparison (acupuncture therapy versus conventional medications) is difficult to blind personnel and participants. The trials also had unclear risk of performance bias for not reporting blinding the outcome assessor. ' Inconsistency; There is significantly statistical heterogeneity indicating by $l^{2}$ value. Imprecision: For dichotomous outcomes, the total number of events is less than 300 , for continu-

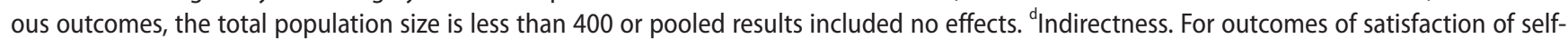
assessment rate, markedly improved on erectile function rate, and erectile angle. This was not internationally applied outcome measures. $\oplus$ : Very low quality of the evidence; $\oplus \oplus$ : Low quality of the evidence.

dence of acupuncture for ED.

There are also some limitations of this systematic review. Firstly, the findings are summarized from original included trials with the poor quality and small sample size, which contributed to reduced internal validity of the pooling result. Secondly, it should be point out that although some combination of acupuncture appeared to show beneficial effect of acupuncture as an adjunctive treatment for ED according to the symptom improvement assessed by IIEF-5 scores scale or self-assessment,our analysis was not able to reach a clear recommendation regarding the effect of acupuncture on ED because most studies showed no benefit of acupuncture alone and variable outcome measure- 
ments used among studies. It is difficult to draw a conclusion addressing the usage of acupuncture in $\mathrm{ED}$ without well-designed trials with a definitive outcome measurement.

\section{Implications for practice}

According to this review, the main acupuncture points used in the treatment of ED were Guan Yuan (CV4), San Yin Jiao (SP6), Shen Shu (BL23), Zu San Li (ST36), Ming Men (DU4), Tai Chong (LR3), Tai Xi (KI3), and Ci Liao (BL32). The stimulation of these acupionts aimed to achieve the needle sensation (de qi) during treatment. In addition, selecting acupionts of abdominal and lumbosacral regions (such as Guan Yuan [CV4], Ci Liao [BL32]) were expected to spread the sensation to the front of the penis or the perineum during the treatment period as well. TCM theory states that ED is usually caused by kidney yang or qi deficiency, the treating acupionts of Shen Shu (BL23), Zhao Hai (KI6) and San Yin Jiao (SP6) were prescribed accordingly [21,23,27,33]. ED may also be caused by other TCM pathophysiological factors such as "the damp-heat pouring downward", the treating acupionts of Qu Quan (LR8), Zhong Wan (RN12), and Yin Ling Quan (SP9) were prescribed accordingly [23,29,33]. However, current clinical evidence is insufficient to support its clinical use. Considering potential therapeutic effects of acupuncture, practitioners may consider its use based clinical experience and preference of patients.

\section{Implications for research}

Considering the variety of acupuncture therapy for $\mathrm{ED}$, future trials should develop optimal acupuncture regimens for ED through Delphi process and/or experts' consensus, validated measurements or tools, such as IIEF-5 scales, to support appropriate interpretation of the findings [11]. In addition, the rational of acupuncture regimen and control should be specified appropriately, and avoid using of comparisons with unclear evidence of effect. To analyze if acupuncture therapy was effective in what kind of ED, the type information of ED participants will be expected to specify clearly in future trials. Furthermore, long-term effect of acupuncture for ED remains unclear. We suggest a followup period should be considered in future trials for the assessment of quality of sexual intercourse or sexual life progression.

To improve the quality of trial design and transpar- ent reporting, we strongly suggest future trials should be prospectively registered on international registry platforms, conducted according to "good clinical practice", and reported according to the Consolidated Standards of Reporting Trials (CONSORT) and Standards for Reporting Interventions in Clinical Trials of Acupuncture (STRICTA) statement [35].

\section{CONCLUSIONS}

Due to insufficient and weak evidence that was summarized, the potential effect of acupuncture as adjunctive treatment (such as compared with tadalafil, psychological therapy or herbal medicine) mainly on psychogenic ED participants is inconclusive and safety of acupuncture was insufficiently reported. Findings of this review should be confirmed in large, rigorously designed and well-reported trial with a definitive outcome measurement.

\section{ACKNOWLEDGEMENTS}

This work is supported by the fund from Beijing University of Chinese Medicine for the Project on Research and Development of Evidence-Based Medicine of Clinical Scientific Research Capacity and International Development in TCM (No.2016-ZXFZJJ-011; No.1000061020008). JP Liu was partially supported by the NCCIH grant (AT001293 with subaward no. 020468C).

\section{Disclosure}

The authors have no potential conflicts of interest to disclose.

\section{Author Contribution}

Conceived of the study: Liu JP. Searched literature, identified studies: Yang XY, Li XL. Extracted data: Li XL, Jia LY, Lai BY. Assessed study quality, analyzed data: Jia LY, Lai BY. Conducted the design of the study and drafted the manuscript: Lai BY. Revised the manuscript: Cao HJ, Yang GY, Grant S, Wong E, Fei YT. Read and approved the final manuscript: all authors.

\section{REFERENCES}

1. Jardin A, Wagner G, Khoury S, Giuliano F, Goldstein I, Padma-Nathan $\mathrm{H}$, et al. Recommendations of the 1st Inter- 
national Consultation on Erectile Dysfunction, co-sponsored by the World Health Organization (WHO). Plymouth: Health Publications Ltd.; 2000.

2. McMahon CG. Erectile dysfunction. Intern Med J 2014;44:1826.

3. Kirby M. The circle of lifestyle and erectile dysfunction. Sex Med Rev 2015;3:169-82.

4. Peak TC, Yafi FA, Sangkum P, Hellstrom WJ. Emerging drugs for the treatment of erectile dysfunction. Expert Opin Emerg Drugs 2015;20:263-75.

5. Tsai MY, Liu CT, Chang CC, Chen SY, Huang ST. Overview of the relevant literature on the possible role of acupuncture in treating male sexual dysfunction. Acupunct Med 2014;32:40610.

6. Kho HG, Sweep CG, Chen X, Rabsztyn PR, Meuleman EJ. The use of acupuncture in the treatment of erectile dysfunction. Int J Impot Res 1999;11:41-6.

7. Shamloul R, Bella A. Complementary and alternative medicine (CAM) for sexual dysfunction. J Sex Med 2014;11:10978.

8. Yin ZH, Ma ZG, Liu CZ. [Study on the mechanism of impotence treated with acupuncture]. J Clin Acupunct Moxib 2004;20:1-2. Chinese.

9. Cui X, Zhou J, Qin Z, Liu Z. Acupuncture for erectile dysfunction: a systematic review. Biomed Res Int 2016;2016:2171923.

10. Jiang R, Qu Y, Wang T, Wan Y, Xu W. Meta-analysis of clinical effectiveness of the acupuncture treatment for erectile dysfunction. Chin J Hum Sex 2018;27:82-5.

11. Rosen RC, Riley A, Wagner G, Osterloh IH, Kirkpatrick J, Mishra A. The international index of erectile function (IIEF): a multidimensional scale for assessment of erectile dysfunction. Urology 1997;49:822-30.

12. Higgins JPT, Green S. Cochrane handbook for systematic reviews of interventions version 5.1.0 [Internet]. London: The Cochrane Collaboration; 2011 [cited 2018 Oct 6]. Available from: http://handbook-5-1.cochrane.org/.

13. Engelhardt PF, Daha LK, Zils T, Simak R, König K, Pflüger $\mathrm{H}$. Acupuncture in the treatment of psychogenic erectile dysfunction: first results of a prospective randomized placebocontrolled study. Int J Impot Res 2003;15:343-6.

14. Liu ZX, Ren ZX. Clinical observation on acupuncture in treatment of erectile dysfunction with type 2 diabetes. Cap Food Med 2015;22:66-7.

15. Li T, Tan Y, Xie ZP, Wang WR, Xie S. [Clinical efficacy observation on moderate-frequency electrical pulse acupoint stimulation combined with tadalafil in treatment of diabetic men with erectile dysfunction]. Chin J Hum Sex 2018;27:213. Chinese.
16. Cui Y, Feng Y, Chen L, Zhou Y, Yang X, He J, et al. [Randomized and controlled research of Chinese drug acupoint injection therapy for erectile dysfunction]. Chin Acupunct Moxib 2007;12:881-5. Chinese.

17. Duan XY. Clinical research of Zhen Yang acupuncture therapy in treating impotence [thesis]. Changchun: Changchun University of Chinese Medicine; 2007.

18. Aydin S, Ercan M, Caşkurlu T, Taşçi AI, Karaman I, Odabaş $\mathrm{O}$, et al. Acupuncture and hypnotic suggestions in the treatment of non-organic male sexual dysfunction. Scand J Urol Nephrol 1997;31:271-4.

19. Chen ZL, Wang GW, Pan PK. [61 cases of psychological erectile dysfunction treated by electro acupuncture combined with Traditional Chinese Herb Medicine]. J Clin Acupunct Moxib 2011;27:43-4. Chinese.

20. Dai EQ, Zhang HW, Liu ZQ. [44 cases of impotence treated with acupuncture combined with Traditional Chinese Medicine]. Shaanxi J Tradit Chin Med 2003;24:66-7. Chinese.

21. Jiang P, Dai N, Yu XH. Therapeutic observation of acupuncture combined with medication for erectile dysfunction. Shanghai J Acupunct Moxib 2014;33:408-9.

22. Jiang XP, Liu XY, Li Q. Clinical observation on effect of acupuncture combined with psychological therapy. Chin Foreign Health Abstr 2012;9:401-2.

23. Lin XL, Deng PH, Yuan JX. Clinical research on treatment of erectile dysfunction with integrative traditional Chinese medicine and acupuncture. Chin J Hum Sex 2005;14:8-10.

24. Liu K, Zeng CH, Ding JY. [Effect of acupoint injection combined with small dose of tadalafil for functional erectile dysfunction]. Modern Tradit Chin Med 2016;36:50-1. Chinese.

25. Liu XJ. Eight liao points warming needle moxibustion in the treatment of impotence 31 cases. Guangming J Chin Med 2017;32:2227-9.

26. Shan YH. Analysis of curative effects of sexual impotence treated by elongated needle and Chinese herb. Shanghai J Acupunct Moxib 2001;2:14-5.

27. Xie DB. Clinical efficacy observation on acupuncture combined with TCM medicine in treatment of male erectile dysfunction. J Hubei College Tradit Chin Med 2016;18:29-31.

28. Ye P, Chen SY. [Observation effect of acupuncture combined with Huan Shao capsule for erectile dysfunction]. J Pract Tradit Chin Med 2017;33:21. Chinese.

29. Jia JB. Clinical observation on the clinical efficacy of acupuncture and moxibustion combined with drug therapy in the treatment of functional ED [thesis]. Wulumuqi: Xinjiang Medical University; 2018.

30. Yu WX, Liu SM, Sun N, Yang LJ, Zhang YY. Clinical observation of press-needling combined with sildenafil in the treat- 
ment of erectile dysfunction. J Clin Acupunct Moxib 2018; 34:27-30.

31. Cao YH, Guo XJ, Cheng YZ. [Treatment of impotence with deficiency of kidney-yang by Chinese medicine and acupuncture]. Tradit Chin Med Res 2007;20:42-3. Chinese.

32. Cheng YZ, Cao YH. [Acupuncture at the point of zhibian (BL54) plus Traditional Chinese Herb Medicine in treating 32 cases of impotence due to deficiency of kidney yang]. Tradit Chin Med Res 2009;22:61-2. Chinese.

33. Ding SL, Zhang FG, Yuan ZJ, Li YF, Qin GZ. [Clinical obser- vation on 88 cases of erectile dysfunction treated by acupuncture combined with medicine]. Yunnan J Tradit Chin Med Materia Medica 2012;33:50-1. Chinese.

34. Yang X, Tian BK. [20 cases of impotence treated with acupuncture combined with Traditional Chinese Herb Medicine]. J Clin Acupunct Moxib 2008;24:27-8. Chinese.

35. Laine C, Horton R, DeAngelis CD, Drazen JM, Frizelle FA, Godlee F, et al. Clinical trial registration: looking back and moving ahead. N Engl J Med 2007;356:2734-6. 


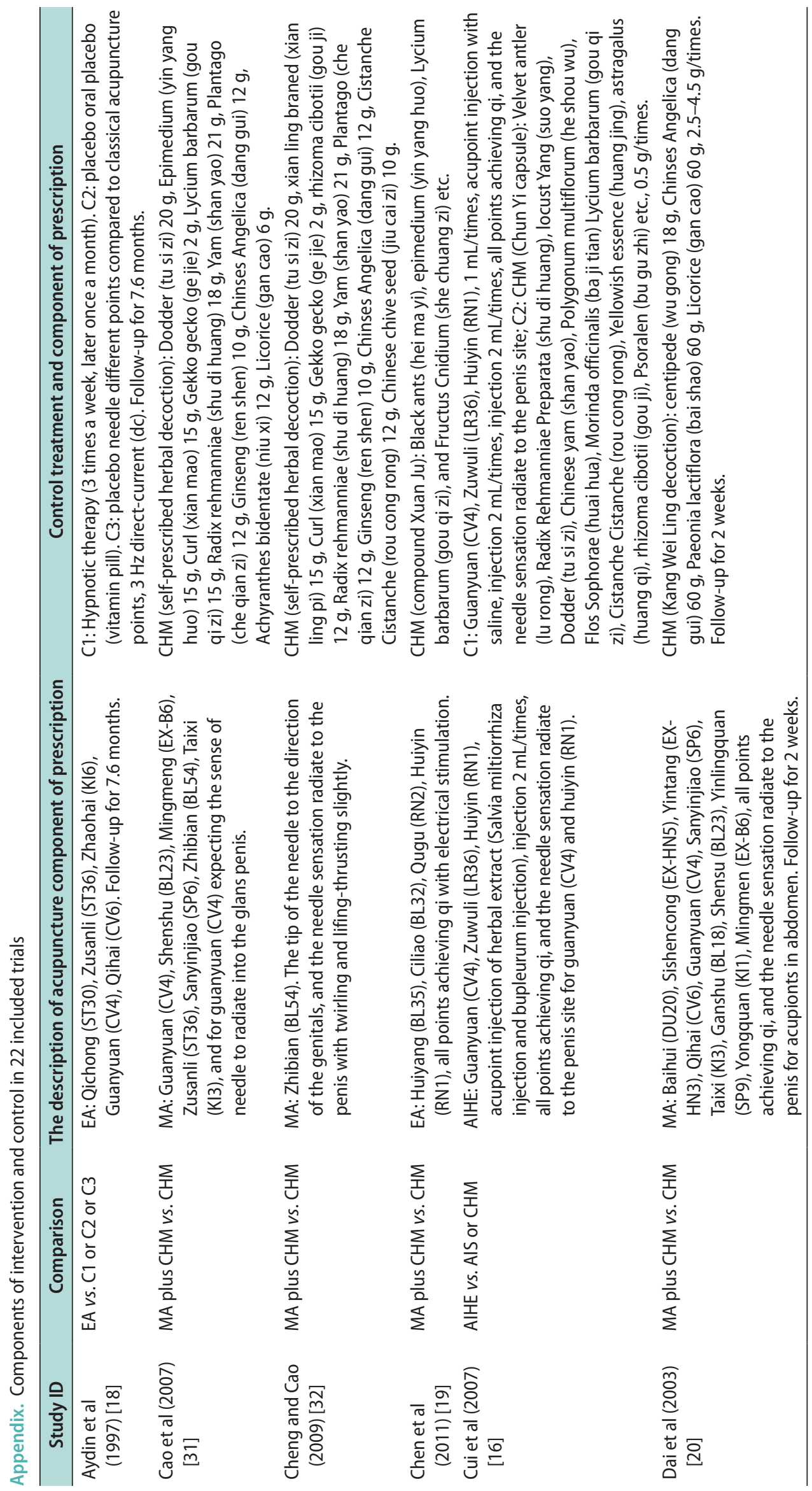




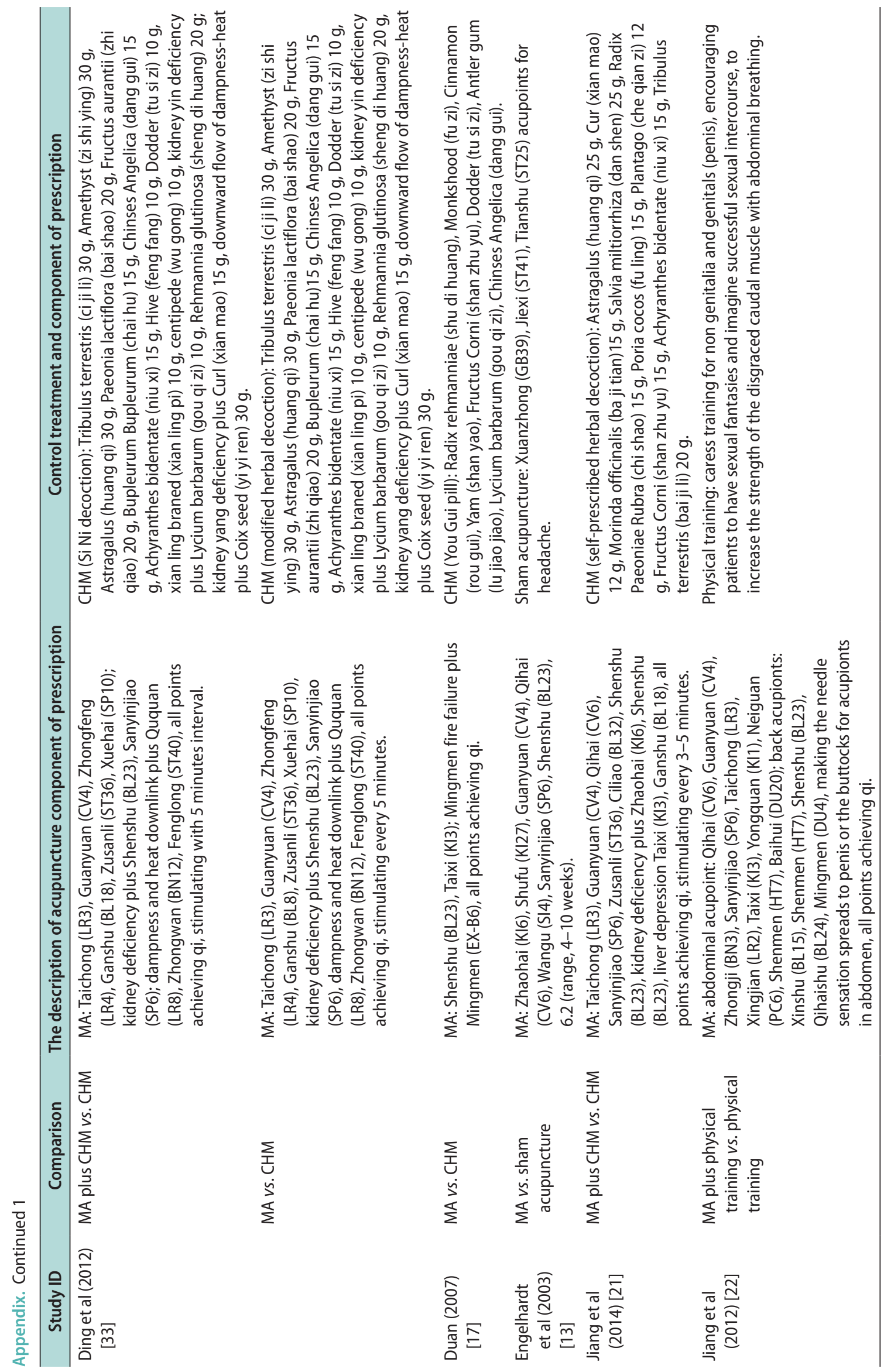




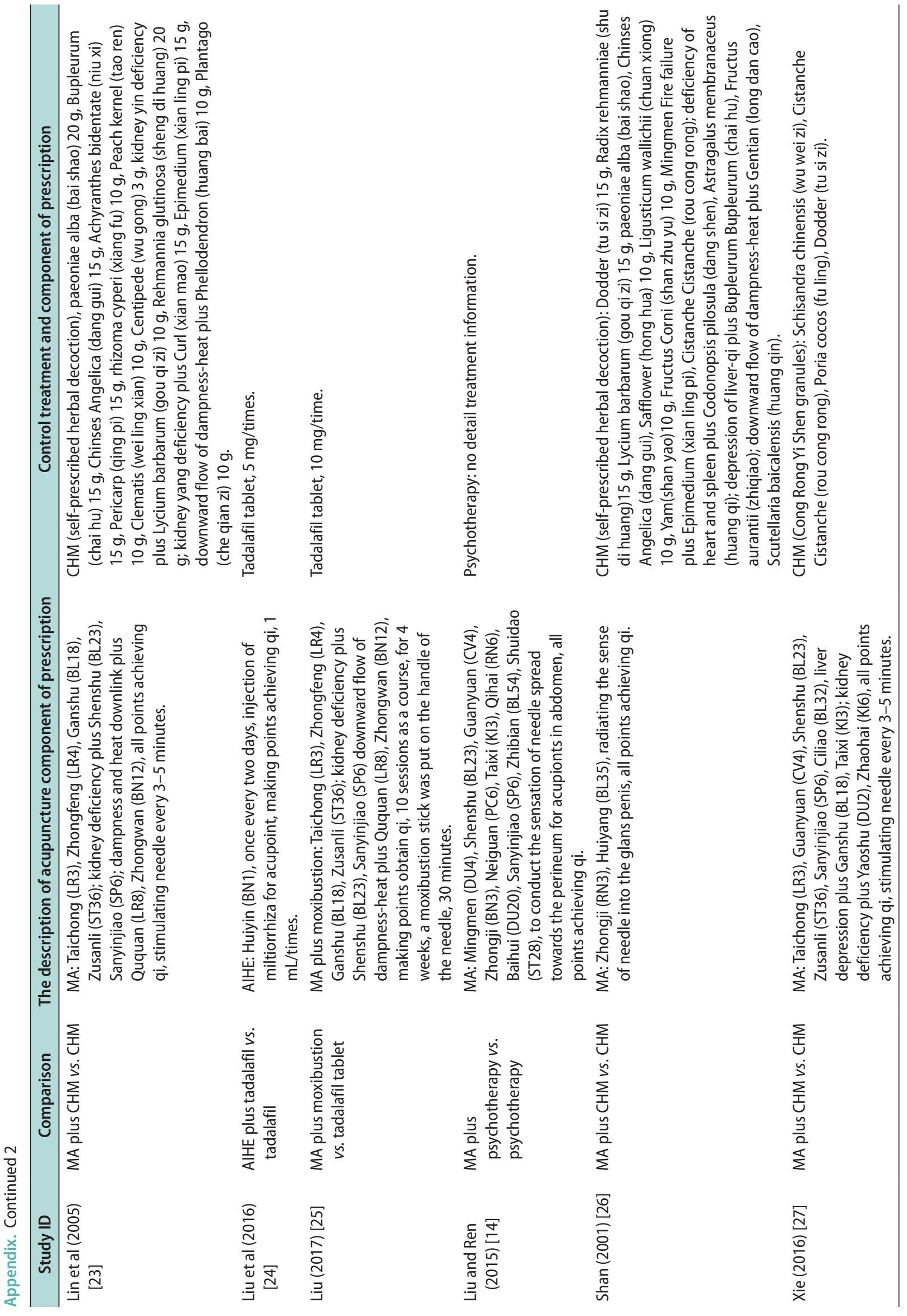




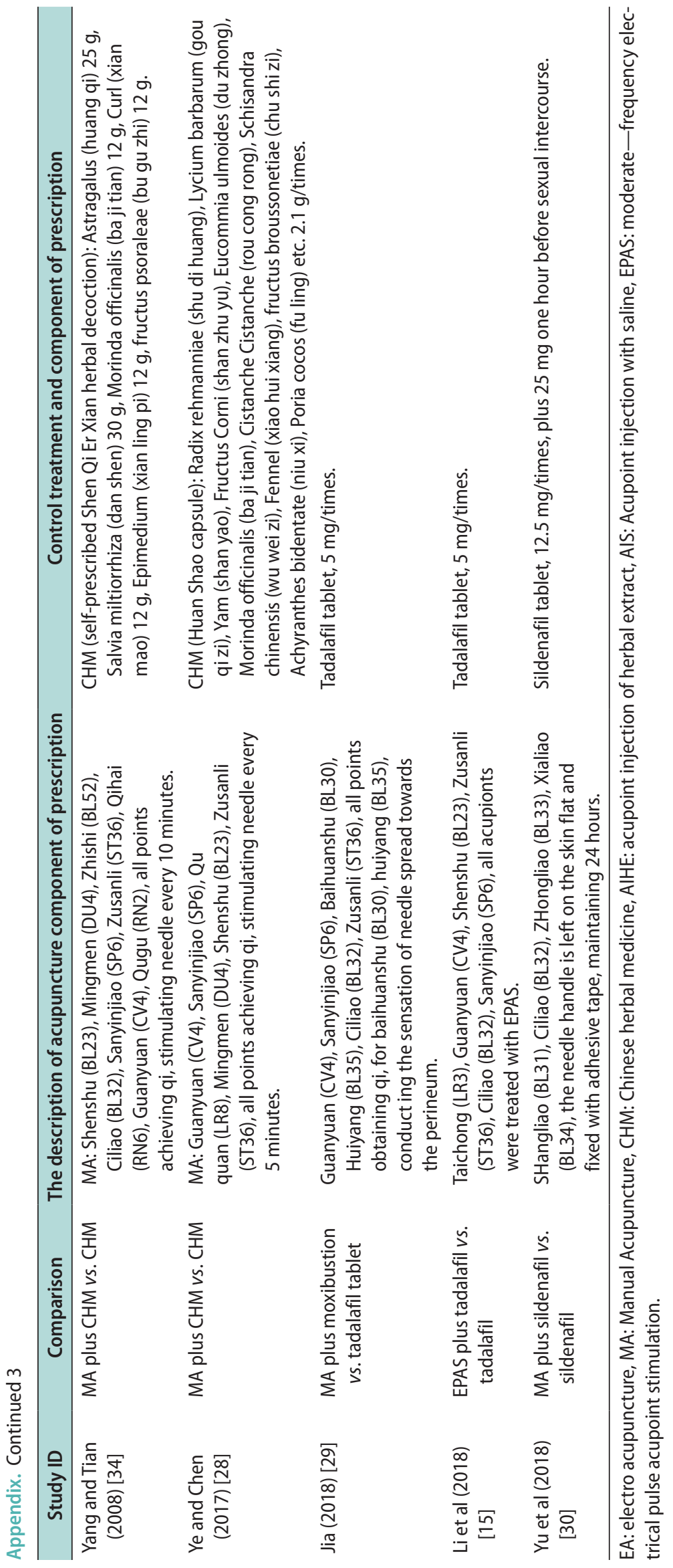

